\title{
Next-generation sequencing of representational difference analysis products for identification of genes involved in diosgenin biosynthesis in fenugreek (Trigonella foenum-graecum)
}

\author{
Joanna Ciura $^{1} \cdot$ Magdalena Szeliga $^{1} \cdot$ Michalina Grzesik $^{1} \cdot$ Mirosław Tyrka $^{1}$ (D)
}

Received: 19 August 2016/Accepted: 30 January 2017/Published online: 4 February 2017

(C) The Author(s) 2017. This article is published with open access at Springerlink.com

\begin{abstract}
Main conclusion Representational difference analysis of cDNA was performed and differential products were sequenced and annotated. Candidate genes involved in biosynthesis of diosgenin in fenugreek were identified. Detailed mechanism of diosgenin synthesis was proposed.

Fenugreek (Trigonella foenum-graecum L.) is a valuable medicinal and crop plant. It belongs to Fabaceae family and has a unique potential to synthesize valuable steroidal saponins, e.g., diosgenin. Elicitation (methyl jasmonate) and precursor feeding (cholesterol and squalene) were used to enhance the content of sterols and steroidal sapogenins in in vitro grown plants for representational difference analysis of cDNA (cDNA-RDA). To identify candidate genes involved in diosgenin biosynthesis, differential, factor-specific libraries were subject to the nextgeneration sequencing. Approximately 9.9 million reads were obtained, trimmed, and assembled into 31,491 unigenes with an average length of $291 \mathrm{bp}$. Then, functional annotation and gene ontogeny enrichment analysis was performed by aligning all-unigenes with public databases.
\end{abstract}

Electronic supplementary material The online version of this article (doi:10.1007/s00425-017-2657-0) contains supplementary material, which is available to authorized users.

\section{Mirosław Tyrka}

mtyrka@prz.edu.pl

1 Department of Biotechnology and Bioinformatics, Faculty of Chemistry, Rzeszow University of Technology, al.

Powstańców Warszawy 6, 35-959 Rzeszów, Poland
Within the transcripts related to sterol and steroidal saponin biosynthesis, we discovered novel candidate genes of diosgenin biosynthesis and validated their expression using quantitative RT-PCR analysis. Based on these findings, we supported the idea that diosgenin is biosynthesized from cycloartenol via cholesterol. This is the first report on the next-generation sequencing of cDNA-RDA products. Analysis of the transcriptomes enriched in low copy sequences contributed substantially to our understanding of the biochemical pathways of steroid synthesis in fenugreek.

Keywords Diosgenin · Next-generation sequencing · Phytosterols - Representational difference analysis of cDNA - Steroidal saponins - Transcriptome user-friendly analysis
Abbreviations
cDNA-RDA Representational difference analysis of cDNA
FPKM Fragments per kilobase of exon per million fragments mapped
GO Gene ontology
KEGG Kyoto Encyclopedia of Genes and Genomes database
KOG Eukaryotic orthologous groups database
MeJ Methyl jasmonate
NR Non-redundant database
RDA-CHL Differential product after cholesterol treatment
RDA-MeJ Differential product after methyl jasmonate treatment
RDA-SQ Differential product after squalene treatment 


\section{Introduction}

Fenugreek (Trigonella foenum-graecum L.) is an annual dicotyledonous legume belonging to the family of Fabaceae. Its genome size is $685 \mathrm{Mbp}$, i.e., approximately $1.5 \times$ larger than the model legumes, Lotus corniculatus $\mathrm{L}$. var. japonicus Regel and barrel (Medicago truncatula Gaertn.), both of which have compact genomes of about $470 \mathrm{Mbp}$ (Vaidya et al. 2013). At present, fenugreek is widely cultivated in India, Argentina, Egypt, and Mediterranean countries. Besides culinary applications, fenugreek leaves and seeds are also consumed for medicinal purposes (Mehrafarin et al. 2011), as they show antidiabetic, hypocholesterolemic, anti-microbial, and anticancer effects. This highly potent female herb helps relaxing the uterus and relieving menstrual pains, is an excellent stimulator of milk production in nursing mothers, and is usually suggested in treatments of poor digestion, gastric inflammations, enteritis, especially for convalescents (Petropoulos 2002; Mehrafarin et al. 2011; Patel et al. 2014).

Fenugreek plants contain active compounds such as alkaloids (e.g., trigonelline, gentianine, choline), flavonoids (e.g., apigenin, luteolin, quercetin, vitexin), steroids (e.g., cholesterol and sitosterol), saponins (e.g., diosgenin, gitogenin, tigogenin), lysine-rich proteins, and volatile oils (Snehlata and Payal 2012). Of these, the most important from pharmacological perspective is diosgenin —a steroidal saponin. Diosgenin is of great interest to pharmaceutical industry as it is used in the treatment of leukemia, hypercholesterolemia, climacteric syndrome, or colon cancer. Diosgenin is used as a substrate for synthesis of oral contraceptives, sex hormones, and other steroidal compounds (Patel et al. 2012). Its anti-cancer activity was proven, and it was found to contribute to an apoptosis of several cancer cell lines (i.e., colon HT-29 and HCT-116, breast AU565 and skin M4Beu) in vitro (Raju and Rao 2012).

Biosynthesis of steroidal saponins, including diosgenin, has not been described in detail yet. Glycolytic, mevalonate, and steroid biosynthesis pathways are involved in diosgenin biosynthesis, and cholesterol was found to be a precursor of this compound (Dewick 2002). It was established that cholesterol is formed from lanosterol and some of these reactions are catalyzed by cytochrome P450 systems (Mehrafarin et al. 2010). Vaidya et al. (2013) reported the first transcriptome sequencing of $T$. foenum-graecum and they concluded that diosgenin might be formed from squalene-2,3-oxide in two ways, (1) from lanosterol via the formation of cholesterol and (2) from cycloartenol via the formation of sitosterol.

The emergence of next-generation sequencing (NGS) has paved the way for large-scale sequencing of industrially important plants. At present, genomes of 39 model and non-model plants have been released and the data are publicly available (http://plants.ensembl.org/index. html). Transcriptomes of Cicer arietinum L., Daucus carota var. sativus L., Hevea brasiliensis, Sesamum indicum L., or Camellia sinensis have been sequenced recently (Annadurai et al. 2012). Sequences of transcriptomes are available also for 14 medicinal plant species (http://medi cinalplantgenomics.msu.edu/index.shtml), and these data linked with metabolic profiles can be exploited to understand regulation and biosynthesis of selected target compounds important for medicinal properties. However, sequencing data for $T$. foenum-graecum are limited.

Efficient methods for profiling cDNA pattern changes in plants showing variable levels of target compounds are prerequisite for discovering new biosynthetic pathways. RNA-seq is a cost-effective, ultra-high-throughput DNA sequencing technology that provides a revolutionary advance in transcriptome-scale sequencing, and is competitive to a microarray technology. Direct sequencing of cDNAs generates short reads that can be assembled into a transcription profile. RNA-seq is a comprehensive and efficient way to qualitatively and quantitatively characterize transcriptomes, and discover new exons and splicing variants (Shi et al. 2011; Chikara et al. 2014). Significant number of medicinal plant species need to be profiled and analyzed for their transcriptomic data to help us (1) understand system biology of their metabolic pathways with reference to the metabolome, (2) compare them across the genes related to biogeneration of the same group of secondary metabolites and with the sets of genes with similar catalytic properties (gene families and super families), (3) elucidate metabolic pathways and their regulation, and (4) understand functional genomics of individual enzymatic step of significance/interest in the species (Sangwan et al. 2013).

There are a number of methods available for subtracting transcripts common for two pools of compared cDNAs. Although these methods are designed to determine qualitative differences between the two pools for up- and downregulated genes, the subtraction should improve frequency of target genes. In this paper, we report on the first time use of NGS of representational difference analysis (RDA) products to discover the genes involved in diosgenin biosynthesis in fenugreek. We used the RDA-NGS approach to sequence the part of fenugreek transcriptome regulated by an elicitor (methyl jasmonate, $\mathrm{MeJ}$ ) and precursors (cholesterol and squalene). The resulting assembled transcripts were functionally annotated, and the transcripts involved in the secondary metabolite biosynthesis pathways, especially of sterols and steroidal saponins, were analyzed. This study improved our understanding of 
molecular signatures in the transcriptome related to physiological functions of plant tissues associated with diosgenin biosynthesis pathway.

\section{Materials and methods}

\section{Plant material}

Seeds of $T$. foenum-graecum were obtained from the Botanic Garden in Bonn (Accession No. 19271). The plants were grown in vitro on Murashige and Skoog (1962) culture medium and were maintained at $25{ }^{\circ} \mathrm{C}$ with a 16 -h light photoperiod. Six-month-old plants were sprayed with 10\%-ethanol solutions of methyl jasmonate (MeJ) $\left(100 \mu \mathrm{L} \mathrm{L}^{-1}\right)$, squalene $\left(100 \mu \mathrm{L} \mathrm{L}^{-1}\right)$, and cholesterol $\left(100 \mathrm{mg} \mathrm{L}^{-1}\right)$. The leaves and stems elicited with MeJ or activated with squalene and cholesterol as precursors were harvested after 3 days, weighed, immediately frozen in liquid nitrogen and stored at $-80{ }^{\circ} \mathrm{C}$ until further analysis. Control plants were sprayed with $10 \%$ solution of ethanol. The experiment was repeated three times. The tissues for analysis were crushed with mortar and pestle in liquid nitrogen. Homogenized samples were used for both RNA isolation and the diosgenin extraction.

\section{Diosgenin extraction and identification}

Solid liquid extraction of diosgenin was performed as described by Savikin-Fodulovic et al. (1998), with modifications. The plant material was hydrolyzed with $1 \mathrm{M}$ sulfuric acid in $70 \%$ isopropanol for $6 \mathrm{~h}$ under reflux. The next step included three cycles of extraction with hexane and then washing with $2 \mathrm{M} \mathrm{NaOH}$ and distilled water. Organic phase was evaporated to dryness on vacuum concentrator at $43{ }^{\circ} \mathrm{C}$.

Diosgenin was identified by ultra performance liquid chromatography (UPLC) coupled with MS with triple quadrupole. A separation was run on C18 RP column (length of $50 \mathrm{~mm}$, internal diameter of $2.1 \mathrm{~mm}$, particle size of $1.7 \mu \mathrm{m})$. The isocratic solvent system was $85 \%$ methanol, flow rate was set at $0.6 \mathrm{~mL} \mathrm{~min}^{-1}$, and the column temperature was maintained at $25{ }^{\circ} \mathrm{C}$. Retention time of diosgenin was $1.87 \mathrm{~min}$. MS was equipped with an electrospray ion source operating in positive ion mode. For targeted metabolites, we used a very sensitive multiple reaction monitoring method. Transition for diosgenin $\mathrm{m} /$ $z 415.3 \rightarrow 271.2$ and $\mathrm{m} / z \quad 415.3 \rightarrow 253.2$ were detected. Measurements were taken in three replicates. Calibration curve was used to measure the concentration of diosgenin. Analysis of variances $(P=0.05)$ was employed to compare the concentrations of diosgenin.

\section{RNA extraction and cDNA library preparation}

Total RNA from plants was isolated by GeneMATRIX Universal RNA Purification Kit (EURx, Gdansk, Poland). The quality and quantity of isolated total RNA were verified on 6000 Nano RNA chip and BioAnalyzer 2100 (Agilent Technologies). Samples with RNA integrity number above 7.5 were used in subsequent steps. Initially, $1 \mu \mathrm{g}$ of total RNA was used for cDNA synthesis. ProtoScript II First Strand cDNA Synthesis Kit and mRNA Second Strand Synthesis Module (New England Biolabs) were used to obtain the first and the second strand of DNA.

\section{Representational difference analysis of cDNA (cDNA-RDA) and sequencing}

To identify up-regulated genes in fenugreek in response to cholesterol, MeJ and squalene representational difference analysis of cDNA was performed. Samples with the highest diosgenin content obtained after treatment with cholesterol, $\mathrm{MeJ}$, or squalene were the testers. Control samples with the lowest diosgenin level were used as drivers. Hubank and Schatz (1994) procedure was followed for cDNA-RDA, with small modifications. Three rounds of subtractive hybridization were carried out, and finally three differential, separate products resulting from the treatment with cholesterol, MeJ, and squalene were obtained. The third round yielded DNA bands and smear in the size range from 200 to $600 \mathrm{bp}$.

Transcriptome libraries for sequencing were constructed as outlined in Illumina's Nextera XT DNA Library Preparation Guide. cDNA libraries were sequenced using paired end Illumina MiSeq. Raw data were saved in FASTQ format.

\section{De novo assembly and functional annotation}

Bioinformatic analysis of raw reads was performed using Transcriptome User-Friendly Analysis (TRUFA; Kornobis et al. 2015), a webserver platform dedicated to RNA-seq analysis. A complete pipeline performs particular tasks in which read files are cleaned and then assembled into transcripts and finally the transcripts are identified and quantified. TRUFA executed the following steps (programs used are specified in the parentheses): reads quality control (FASTQC); quality trimming and duplicate removal (Prinseq); filtering out potential contaminants (Blat); de novo assembly of reads (Trinity); reads mapping (Bowtie2); contigs (i.e., transcripts) identification based on sequence alignment (Blat, Blast), protein dominions, profiles (HMMER) and annotation with gene ontology (GO) terms (Blast2GO); expression quantification. 
For non-redundant protein database (NR) annotation, the Blast2GO PRO program (Conesa et al. 2005) was applied to obtain GO annotations according to molecular function, biological process, and cellular component ontologies. Each annotated sequence may have more than one GO term, either assigned in different GO categories (biological process, molecular function, and cellular component) or in the same category. Enzyme commission number was assigned and parsed based on the BLAST2GO results.

Additionally, unigenes ( $\geq 201 \mathrm{bp}$ ) were annotated by aligning against Swiss-Prot database by BLASTX $(e$ value $\leq 1 e-5)$ and EuKaryotic Orthologous Groups (KOG) databases using WebMGA server (Wu et al. 2011). WebMGA performs function annotation using RPSBLAST program on KOG database (eukaryotic proteins). Assembled sequences were also submitted to the online Kyoto Encyclopedia of Genes and Genomes database (KEGG) Automatic Annotation Server (KAAS) with bi-directional best hit method (Moriya et al. 2007).

\section{Validation of transcripts by qRT-PCR}

Selected unigenes, annotated to sterol and sapogenin biosynthesis, were chosen for validation using real-time qPCR with gene-specific primers designed with PrimerBlast (Ye et al. 2012). One microgram of total RNA was converted to cDNA using M-MuLV (Moloney Murine Leukemia Virus) Reverse Transcriptase (EURx) by using oligo(dT) primers. Primers for the seven selected unigenes were designed (Table S1). The reaction was performed using GoTaq qPCR Master Mix kit (Promega) on Eco Real-Time PCR System (Illumina) using the following program: $95{ }^{\circ} \mathrm{C}$ for $10 \mathrm{~min}$ and 40 cycles of $95{ }^{\circ} \mathrm{C}$ for $15 \mathrm{~s}$, anneal at $60{ }^{\circ} \mathrm{C}$ for $60 \mathrm{~s}$.

The actin gene was selected as an internal standard for normalization, and three biological and two technical replicates were completed for each gene. Relative expression levels for each unigene were calculated using a deltadelta $C_{\mathrm{t}}\left(2^{-\Delta \Delta C_{\mathrm{t}}}\right)$ method. All data were expressed as the mean $\pm \mathrm{SD}$ after normalization (Livak and Schmittgen 2001).

\section{Results}

\section{Diosgenin content}

Fenugreek plants were treated with methyl jasmonate (MeJ), cholesterol, and squalene to activate the genes involved in biosynthesis of diosgenin. Diosgenin content was determined in fresh plants after the treatments vs control (Fig. S1). Differences between samples were statistically significant. In respect to control $\left(36.7 \mu \mathrm{g} \mathrm{g}^{-1}\right)$, higher concentration of diosgenin in fresh plants was observed after treatment with $\mathrm{MeJ}\left(359 \mu \mathrm{g} \mathrm{g}^{-1}\right)$, squalene (243 $\mu \mathrm{g} \mathrm{g}^{-1}$ ), and cholesterol (186 $\left.\mu \mathrm{g} \mathrm{g}^{-1}\right)$. Methyl jasmonate is a well-known compound that affects signal transduction involved in the elicitation process. The positive effect of MeJ on diosgenin production was previously reported (De and De 2011), and $\mathrm{MeJ}$ at $100 \mu \mathrm{L} \mathrm{L}^{-1}$ enhanced diosgenin content by 10.5 times. Cholesterol was applied for an induction of steroidal saponins (Aasim et al. 2014), but no information about the use of squalene was available.

\section{Sequencing and de novo assembly}

Three final products of subtraction of cDNA pools (RDACHL, RDA-MeJ, and RDA-SQ) were sequenced on Ilumina MiSeq sequencer. Double-strand sequencing of T. foenum-graecum transcriptomes resulted in a total number of $9,918,886$ reads $(3,035,834$ for RDA-CHL; 3,674,418 for RDA-MeJ; and 3,208,634 for RDA-SQ) with an average read length of $301 \mathrm{bp}$. The sequences were filtered by removal of duplicates and potential contaminants, and then quality-filtered sequences were submitted for de novo assembly. Cleaning and filtering reduced the number of sequences by about $33 \%$ to $1,036,467$, $1,239,927$, and 1,059,468 for RDA-CHL, RDA-MeJ, and RDA-SQ, respectively. De novo assembly was performed using Trinity (Grabherr et al. 2011) and reads were mapped through Bowtie2 (Langmead and Salzberg 2012) with default parameters implemented by TRUFA (Kornobis et al. 2015). A total of 11,603 contigs for RDA-CHL, 9029 for RDA-MeJ, and 10,859 contigs for RDA-SQ were generated (Table 1). An average length of contigs amounted to 290 bases, and only $2 \%$ of the sequences were longer than $500 \mathrm{bp}$. The transcripts were rich (about 57\%) in AT nucleotides.

Table 1 Summary of sequences assembly

\begin{tabular}{llll}
\hline & RDA-CHL & RDA-MeJ & RDA-SQ \\
\hline Total number of contigs & 11,603 & 9029 & 10,859 \\
Min length (bp) & 201 & 201 & 201 \\
Max length (bp) & 843 & 730 & 743 \\
Average length (bp) & 293.30 & 287.94 & 292.91 \\
Total bases in contigs (bp) & $3,403,105$ & $2,599,813$ & $3,180,692$ \\
Number of contigs $<500$ bp & 11,409 & 8935 & 10,673 \\
Number of contigs $\geq 500$ bp & 194 & 94 & 186 \\
N50 (bp) & 295.0 & 289.0 & 295.0 \\
Contigs in N50 & 4710 & 3730 & 4369 \\
GC content $(\%)$ & 42.46 & 43.45 & 42.55 \\
\hline
\end{tabular}




\section{Functional annotation}

Functional annotation of novel plant transcriptomes is a challenging task due to limited availability of reference genome/gene sequences in public databases (Annadurai et al. 2012). In non-model fenugreek plant, homologies with reference legumes (L. corniculatus and M. truncatula) are expected. Annotation of fenugreek transcriptome was based on sequence-based alignments. For this purpose, we performed a search against five public databases, including the NR, UniProt Reference Clusters (UniRef90), Swiss-Prot, KEGG, and KOG (Table 2). The highest number of hits (from $90.4 \%$ for RDA-CHL to $91.5 \%$ for RDA-MeJ) was found for NR and UniRef90 databases. Blast search against Swiss-Prot database revealed homologies for about $62 \%$ of unigenes.

\section{Gene ontology (GO) classification}

Based on NR annotations, GO analysis was performed to distribute unigenes into three major categories of cellular component, molecular function, and biological process. Annotated sequences may have variable number GO terms representing different categories. For all the unigenes selected in the RDA procedure, we found Medicago truncatula, C. arietinum, and Glycine max as the top-hit species with the highest distribution of similar sequences (Table S2). About $71 \%$ of transcripts within RDA pools were annotated with GO terms.

Distribution of GO terms between the three subtracted libraries was similar. Majority of the unigenes (53\%) were annotated to biological processes, followed by molecular functions (27\%) and cellular components (20\%). In the biological processes category, the classes related to metabolic process (22\%, GO:0008152), cellular process (20\%, GO:0009987), single-organism process (17\%, GO:0044699), biological regulation (7\%, GO:0065007), and response to stimulus (6.7\%, GO:0050896) were the most frequently observed. In molecular functions class, catalytic activity (45\%, GO:0003824) and binding (42\%, GO:0005488) were found to be the most abundant sub- categories. The most common GO terms within cellular components included a cell (36\%, GO:0005623), organelle (26\%, GO:0043226), and membrane (16\%, GO:0016020).

\section{Multilevel GO analysis for biological process ontologies to trace secondary metabolism}

The role of all the unigenes in secondary metabolism and sterol biosynthesis was analyzed using multilevel procedure (Table S3). At the third level, over 2300 of unigenes were involved in biosynthetic process and most of them played a role in the metabolism of small molecules and organic cyclic compounds. High number of sequences participated in the metabolic process of isoprenoid, terpenoid, and saponin biosynthesis. When compared to other RDA products, RDA-SQ transcripts were more abundant in steroid and sterol metabolic processes, and in biosynthesis of steroids, phytosterols, and sterols.

\section{Enzyme commission classification}

Depending on RDA pool, from 2667 to 3277 unigenes were classified as enzymes, and could be further divided into six subclasses. The most frequently represented enzymes were transferases (34.6-36.2\%), hydrolases (25.2-26.2\%), and oxidoreductases (20.9-22.3\%). Ligases, lyases, and isomerases accounted for $3.6-7.8 \%$ of the enzymes, depending on RDA pool (Fig. 1).

The most frequently detected enzymes belonging to enzyme commission sub-categories for each RDA transcripts were 2.7, transferring phosphorus-containing groups (17.9\% for RDA-CHL, $16.2 \%$ for RDA-MeJ, and $17.4 \%$ for RDA-SQ); 3.1, acting on ester bonds (7.1, 6.4, and $7.3 \%$ for RDA-CHL, RDA-MeJ, and RDA-SQ, respectively); 2.4, glycosyltransferases $(6.2,5.8$, and $6.4 \%$ for RDA-CHL, RDA-MeJ, and RDA-SQ, respectively); 3.2, glycosylases $(6.1,6.2$, and $6.2 \%$ for RDA-CHL, RDA-MeJ, and RDA-SQ, respectively); and 3.4, acting on peptide bonds (peptide hydrolases) (6.0, 6.2, 6.0\% for RDA-CHL, RDA-MeJ, RDA-SQ, respectively).
Table 2 Summary of annotations of the T. foenumgraecum unigenes

\begin{tabular}{lllr}
\hline & \multicolumn{2}{l}{ Number of sequences } & \\
\cline { 2 - 4 } & RDA-CHL & RDA-MeJ & RDA-SQ \\
\hline All assembled unigenes & 11,720 & 9080 & 10,942 \\
Gene annotations against NR & 10,598 & 8316 & 9933 \\
Gene annotations against UniRef90 & 10,467 & 8207 & 9827 \\
Gene annotations against Swiss-Prot & 7251 & 5754 & 6782 \\
Gene annotations against KOG & 5610 & 4583 & 5311 \\
Gene annotations against KEGG & 3123 & 2577 & 3001 \\
Gene annotations against Pfam & 9013 & 7104 & 8445 \\
\hline
\end{tabular}


Fig. 1 Enzyme commission classification of transcripts for different RDA pools

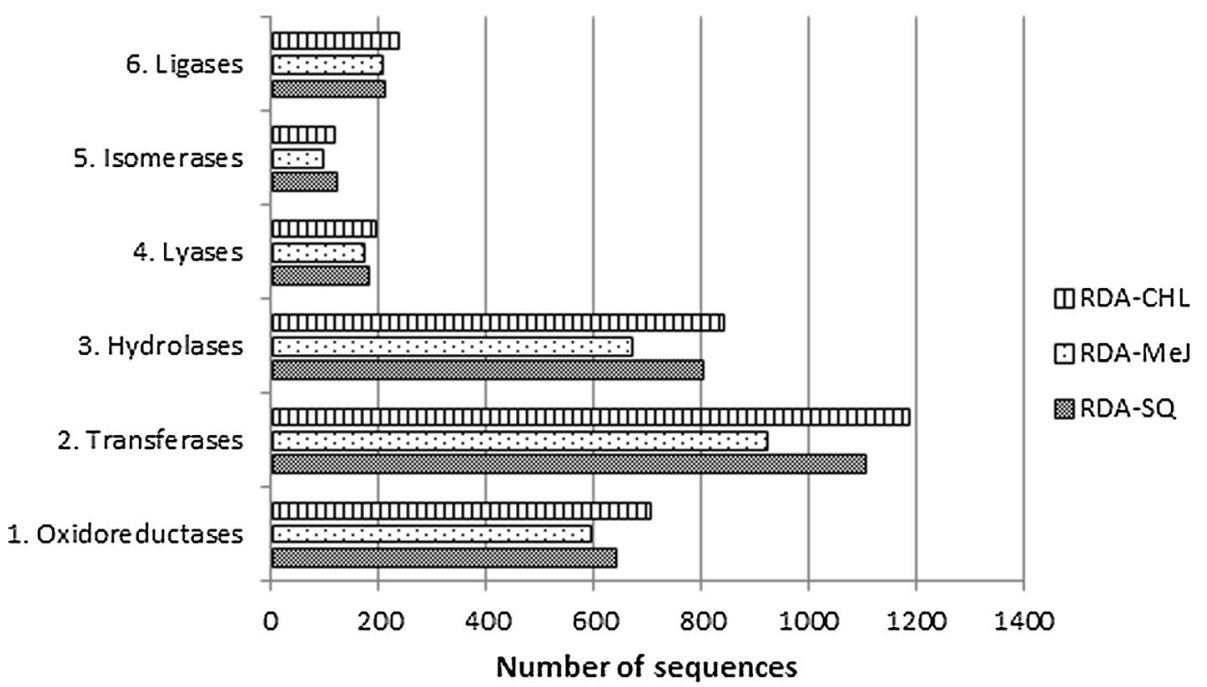

\section{Kyoto Encyclopedia of Genes and Genomes (KEGG) pathway mapping}

To identify the biological pathways that are active in fenugreek, all the assembled sequences were assigned with KEGG orthology (KO) identifiers using KEGG Automatic Annotation Server (KAAS) with the bi-directional best hit information method. We focused on steroid and terpenoid pathways to identify the enzymes involved in steroidal sapogenin biosynthesis. As a result of KEGG annotations, 3123, 2577, and 3001 sequences were assigned for RDACHL, RDA-MeJ, and RDA-SQ, respectively. In selected KEGG sub-categories, the most frequently represented pathways were those related to ribosome, spliceosome, protein processing in endoplasmic reticulum, RNA transport, starch and sucrose metabolism, and glycolysis/gluconeogenesis (Table S4).

Two hundred and sixteen unigenes in RDA-CHL, 187 in RDA-MeJ, and 210 unigenes in RDA-SQ were significantly matched to $26 \mathrm{KEGG}$ pathways related to secondary metabolite biosynthesis (Table S5). We found 36, 35, and 43 unigenes encoding enzymes involved in steroid and terpenoid backbone biosynthesis in RDA-CHL, RDA-MeJ, and RDA-SQ libraries.

\section{Classification at Eukaryotic Orthologous Groups database (KOG)}

The KOG proteins from the eukaryotic clusters were used to annotate 5610 transcripts for RDA-CHL ( $47.87 \%$ of all transcripts), 4583 for RDA-MeJ (50.47\%), and 5311 for RDA-SQ (48.54\%) libraries (Table S6). Among 25 KOG categories, the three largest clusters for each RDA transcripts were signal transduction mechanisms $(717,545$, and 659 counts for RDA-CHL, RDA-MEJ, and RDA-SQ, respectively), posttranslational modification, protein turnover, chaperones (698 RDA-CHL, 613 RDA-MeJ, 704 RDA-SQ), and general function prediction only (697 RDACHL, 562 RDA-MeJ, 667 RDA-SQ). The categories of defense mechanisms, nuclear structure, extracellular structures, and cell motility were represented by the smallest groups of unigenes. The category of secondary metabolites biosynthesis, transport, and catabolism was represented by about $4 \%$ of genes corresponding to 261 , 211, and 234 transcripts from RDA-CHL, RDA-MeJ, and RDA-SQ libraries, respectively. The most abundant sequences in this category were those of cytochrome P450 with a total 79 unigenes for RDA-CHL (30.2\%), 66 unigenes for RDA-MeJ (31.3\%), and 68 unigenes for RDA-SQ (29\%). Additionally, for each RDA product, the most frequent sequences were found for iron/ascorbate family oxidoreductases, alcohol dehydrogenases, multidrug resistance-associated proteins/mitoxantrone resistance proteins, $\mathrm{ABC}$ superfamily, and pleiotropic drug resistance proteins (PDR1-15).

\section{Pfam annotations}

Searching against the Pfam database using profile hidden Markov model methods resulted in annotations of 9013, 7104, and 8445 transcripts for RDA-CHL, RDA-MeJ, and RDA-SQ selected sequences, respectively. The aim of this approach was to identify similarities at domain level. The proteins that have little similarity at nucleotide sequence level might share conserved structural domains. Based on the frequency of the occurrence of transcripts in each Pfam domain, we ranked the Pfam domains/families and listed the fifteen top abundant domains/families (Table S7).

Among Pfam annotated sequences, protein kinase domain and its sub-class protein tyrosine kinase were the most frequently represented. These domains regulate a majority of cellular pathways (Zhang et al. 2015). Next 
most abundant sequences contained WD40 domain that is important for signal transduction mechanisms (Annadurai et al. 2012). The other frequently occurring domains in RDA products were cytochrome $\mathrm{P} 450$ (that mediates oxidation of organic substances and plays a significant role in secondary metabolite biosynthesis) and major facilitator superfamily (a class of membrane transport proteins that facilitate movement of small solutes across cell membranes). Other protein domains like RNA recognition motif, zinc finger, or leucine-rich repeats were also found.

\section{Identification of transcripts related to terpenoid backbone biosynthesis}

Terpenoid backbone is formed from condensation of fivecarbon building blocks designated as 3-isopentenyl pyrophosphate (IPP, C5) and dimethylallyl pyrophosphate (DMAPP, C5). In plants, IPP and DMAPP either derive from cytosolic mevalonate pathway via condensation of acetyl-CoA or from pyruvate and phosphoglyceraldehyde in the plastidial non-mevalonate pathway (MEP) (Augustin et al. 2011). In the next step, three isoprene units are linked to each other in a head-to-tail manner, resulting in a 15 C-atom molecule of farnesyl pyrophosphate (FPP). Two farnesyl pyrophosphates are subsequently linked in a tailto-tail manner to give squalene, which is subsequently epoxygenated to 2,3-oxidosqualene. 2,3-oxidosqualene is considered a precursor of triterpenoid saponins, phytosterols, and steroidal saponins (Vincken et al. 2007; Xue et al. 2012). Two important pieces of information on this pathway are that mevalonate is not an intermediate in the plastidial synthesis of IPP and DMAPP, and that the MEP pathway appears to be largely responsible for the biosynthesis of monoterpenes, diterpenes, tetraterpenes (carotenoids), and polyprenols. Sesquiterpenes, sterols, and triterpenes are synthesized in the cytosol via the mevalonate pathway, which is responsible for the synthesis of the key intermediate FPP (Chappell 2002).

We found 34, 25, and 31 unigenes related to terpenoid backbone biosynthesis in RDA-CHL, RDA-MeJ, and RDA-SQ libraries, respectively (Table S8). Almost all of the genes encoding the enzymes involved in this pathway were present in our data. It was suggested that 3-hydroxy3-methylglutaryl-CoA reductase (HMGR), squalene synthase (SQS), and squalene epoxidase (SQE) enzymes of the mevalonate pathway represented the rate-limiting or regulatory enzymes for saponin biosynthesis (Hwang et al. 2015). We identified 12, 5, and 4 non-redundant unigenes corresponding to HMGR, SQS, and SQE, respectively. Single or multiple unigenes were assigned to the enzymes involved in terpenoid backbone biosynthesis. These unique sequences could be assigned to different members of a gene family, different fragments of a selected gene, or both (Upadhyay et al. 2014).

\section{Identification of transcripts related to cholesterol biosynthesis and phytosterol pathway}

Sterols are isoprenoid-derived molecules that have essential functions in eukaryotes in general, and in higher plants in particular. In vertebrates, cholesterol is by far the major sterol, whereas a mixture of various sterols is present in higher plants: sitosterol (usually predominant), stigmasterol, campesterol, and other. In plants, sterol biosynthesis pathway yields 24-methyl- and 24-ethylsterols. A number of minor sterols were detected suggesting that in addition to the main sterol biosynthesis pathway depicted here a number of side pathways existed (Benveniste 2004).

In the pathway including cholesterol, we identified $\Delta^{14}$ sterol reductase (EC:1.3.1.70), sterol-4 $\alpha$-methyl oxidase (EC:1.14.13.72), $\Delta^{24}$-sterol reductase (EC:1.3.1.72), cholestenol delta-isomerase (EC:5.3.3.5), and $\Delta^{7}$-sterol-C5(6)desaturase (EC:1.14.21.6). For phytosterol biosynthesis via a sitosterol, we found cycloartenol synthase CAS (5.4.99.8), sterol 24-C-methyltransferase SMT1 (EC:2.1.1.41), cycloeucalenol cycloisomerase CPI (EC:5.5.1.9), sterol 14-demethylase CYP51 (EC:1.14.13.70), $\Delta^{14}$-sterol reductase FK (EC:1.3.1.70), $\Delta^{8}-\Delta^{7}$-sterol isomerase HYD1 (EC:5.3.3.5), 24-methylenesterol C-methyltransferase SMT2 (EC:2.1.1.143), sterol-

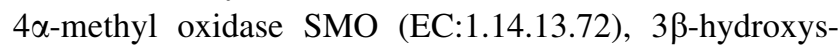
teroid-4 $\alpha$-carboxylate 3-dehydrogenase HSD (1.1.1.-), $\Delta^{7}$ sterol-C5(6)-desaturase DWF7 (EC:1.14.21.6) 7-dehydrocholesterol reductase DWF5 (EC:1.3.1.21), $\Delta^{24}$-sterol reductase DWF1 (EC:1.3.1.72), and cytochrome CYP710A (Table S8 and S9).

\section{Validation of selected transcripts by qRT-PCR}

To examine the expression pattern of genes encoding the enzymes involved in steroid biosynthesis pathways, we used qRT-PCR. Fragments per kilobase of exon per million fragments mapped (FPKM) values from expression quantification algorithm are the values used to measure relative expression of a transcript. In our study, the FPKM ranged from 0 to 4809.92, which indicated that unigenes showed a wide range of expression level (Table S9). We compared the values of FPKM and sequences of unigenes from RDACHL, RDA-MeJ, and RDA-SQ for individual enzymes involved in sterol and diosgenin biosynthesis. Seven unigenes were selected and RT-PCR was successfully applied to amplify unique products of the expected sizes. Based on the delta-delta $C_{\mathrm{t}}\left(2^{-\Delta \Delta C_{\mathrm{t}}}\right)$ method, relative expression 
levels of the selected unigenes were calculated and compared among the three differential products.

All the transcripts showed minor differences in relative quantification RQ (Fig. 2). Increased expression was identified for CYP18A1, CYP734A1, and unspecific monooxygenase. The greatest differences between cDNARDA products were determined for CYP18A1 and unspecific monooxygenase EC:1.14.14.1.

For three selected unigenes, high FPKM values were not supported by RQ scores (Fig. 3). For CPI FPKM, the RQ values were the highest for RDA-SQ and RDA-CHL, respectively. Similar results were obtained for unspecific monooxygenase. For CYP18A1, the highest FPKM was related with RDA-CHL and similar value was found for RQ.

Overall, the results of qRT-PCR experiments were in agreement with FPKM data, and confirmed that the selected unigenes were efficiently induced in cholesterol-, $\mathrm{MeJ}$-, and squalene-treated samples, and that sequencing data obtained from the assembled transcriptome were reliable.

\section{Discussion}

\section{Induction of diosgenin biosynthesis in vitro}

Synthesis of secondary metabolites is often stimulated by biotic or abiotic stresses. Saponins exhibit a wide range of biological activities and take part in mechanisms of defense and protection as well as in environmental interactions (Szakiel et al. 2011). Saponins are used to defend plant against pathogens. Upon a pathogen infection, saponin content increases as a result of chemical modifications of their precursor molecules. Another role of saponins in plants is to protect them against herbivores and/or insects and this is mostly based on their acting as deterrents, toxins, and digestibility inhibitors. This is associated with their capacity to disrupt cell membranes (Faizal and Geelen 2013).

Plant cell and tissue culture systems represent a potential source of valuable secondary metabolites. A production in in vitro cultures is not dependent on geography and season, product quality and yield are generally established, and clonal propagation methods can be used to overcome germination and plant heterogeneity issues. In addition, improved production of desired compounds is easily achievable in in vitro cultures using biotechnological methods (Lambert et al. 2011). Plant cells in vitro show physiological and morphological responses to microbial, physical, or chemical factors that are known as elicitors. Elicitation is an induced or enhanced biosynthesis of metabolites triggered by an addition of trace amounts of elicitors (Smetanska 2008). These defense responses can be activated through a signal transduction pathway via recognition of an "elicitor" by specific receptors located in the plasma membrane and formation of secondary messengers, such as jasmonates, ethylene, and salicylic acid, which in turn activate the expression of defense genes, including the genes that encode the enzymes catalyzing the formation of secondary metabolites (Lambert et al. 2011). Numerous examples of elicitors that enhance saponin production can be found in research literature.

Upadhyay et al. (2014) studied transcriptional changes in leaf and root tissues of Asparagus racemosus after methyl jasmonate treatment. They found that the transcripts of UGTs and CYP450 involved in glycosylation and oxygenation steps were accumulated following the treatment. This up-regulation might be due to the fact that mono-oxygenases that catalyze oxygenation reactions and glycosyltransferases that catalyze the transfer of sugar molecules to steroidal compounds may produce diverse
Fig. 2 Relative expression levels of secondary metabolismrelated gene transcripts. Vertical bars denote \pm SD. CPI cycloeucalenol cycloisomerase, CYP51 sterol 14-demethylase, DWF5 7-dehydrocholesterol reductase, $D W F 1$ sterol $\triangle 24$ reductase, CYP18A1 26-hydroxylase, $C Y P 734 A 1$ 26-hydroxylase, EC:1.14.14.1 unspecific monooxygenase

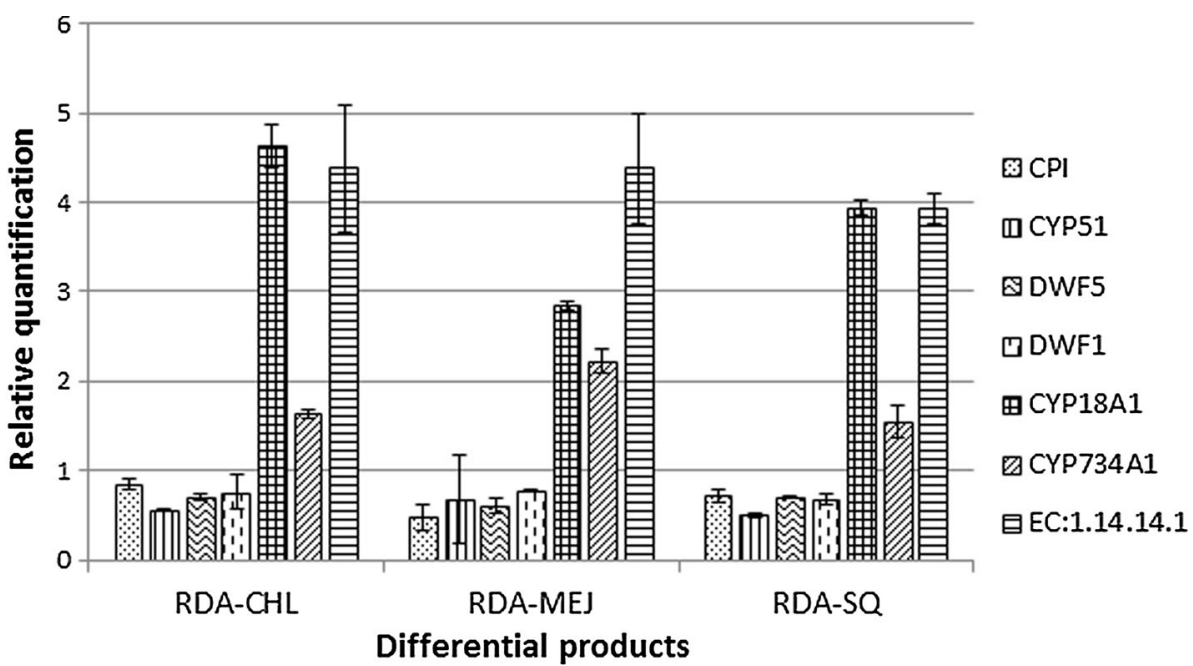



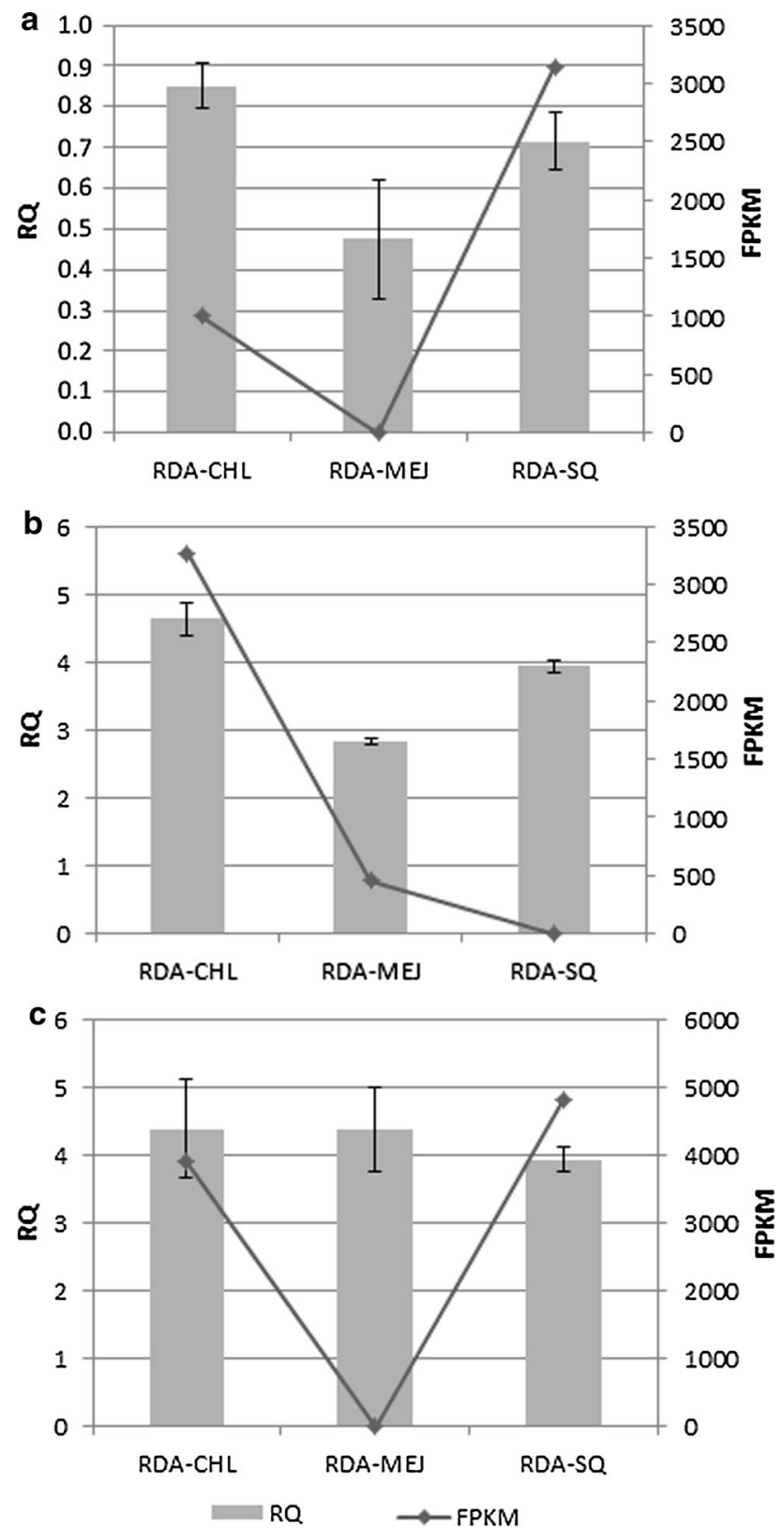

Fig. 3 Quantitative qRT-PCR validations of cycloeucalenol cycloisomerase (a), CYP18A1 (b), and unspecific monooxygenase (c)

saponins under different conditions (stress) and control plant activities. Similarly, in Eleutherococcus senticosus, siberian ginseng, which was exposed to MeJ, the genes involved in saponin biosynthesis pathway that might be involved in triterpene formation, hydroxylation, or oxidation of triterpene skeletons by CYP and glycosylation by UGT were up-regulated (Hwang et al. 2015). Recently, Cao et al. (2015) found that MeJ treatment up-regulated squalene synthase, squalene epoxidase, and dammarenediol synthase in Panax ginseng. Also in fenugreek, the application of MeJ caused an increase in diosgenin level and enhanced the expression of 3-hydroxy-3-methylglutaryl-
CoA reductase (HMGR) and sterol-3-glucosyltransferase genes involved in the biosynthetic pathway of saponins (Chaudhary et al. 2015). Application of methyl jasmonate induced the genes involved in saponin biosynthesis and accumulation. Our results confirmed the effectiveness of MeJ treatment in enhancing the production of diosgenin in fenugreek tissues.

\section{Next-generation sequencing of cDNA-RDA products}

Representational difference analysis is a powerful and sensitive tool for the identification of differentially expressed genes in two different cDNA populations and it allows for detection of changes in mRNA expression by selective enrichment without any prior knowledge of the gene in question (Leite et al. 2013). Moreover, cDNARDA is capable of detecting both relative (greater than three- to five-fold) and absolute differences in expression (Hubank and Shatz 1999). This very high sensitivity and specificity of cDNA-RDA approach should allow the genes switched on, or up- or down-regulated, in both the treated sample and its control, to be analyzed in the same experiment (Bowler et al. 1999). In our study, comparison of samples with contrasting levels of diosgenin content made it possible to identify the genes involved in steroidal saponin biosynthesis. Application of RDA procedure to reduce the amount of overrepresented transcripts partially compensates lower sequencing resolution of Miseq when compared to Hiseq.

In this study, we sequenced RDA products and obtained a total of 31,491 unigenes (11,603 for RDA-CHL; 9029 for RDA-MeJ; and 10,859 for RDA-SQ) by de novo assembly. These unigenes were then annotated in different databases under various criteria like gene or protein name descriptions, conserved domains, GO terms, and metabolic pathways. Detailed functional information is very important to understand overall expression profiles and known biosynthetic pathways of secondary metabolites. The most annotated unigenes were found in NR and UniProt Reference Clusters 90 databases.

Methyl jasmonate stimulates defense reaction of plants to biotic and abiotic stresses. Cholesterol and squalene are expected to participate mainly in metabolism of terpenoids and sterols. Therefore, more unigenes can be expected after MeJ treatment, compared to cholesterol or squalene. However, sequencing of differential products revealed less unigenes after $\mathrm{MeJ}$ treatment than following the treatments with cholesterol and squalene (Tables S2-S5). The quantities of normalized cDNA libraries used for sequencing were equimolar and the yields of raw data were similar. If the targeted genetic change does not result in a unique DNA fragment after digestion, then the change cannot be detected (Chang 2003). Thus, the discrepancies in the 
number of unigenes can be explained by the nature of RDA procedure that equilibrates the levels of overexpressed transcripts and favors diversity of cDNA fragments present at low levels.

We found that four down-regulated genes (CPI, CYP51, DWF5, and DWF1) belonged to the cycloartenol to cholesterol pathway, and three up-regulated transcripts (CYP18A1, CYP734A1, and EC:1.14.14.1) represented cholesterol to diosgenin pathway. Activity of the last three genes may be directly connected with elevated levels of diosgenin (Fig. S1). This observation suggests that both pathways (leading to cholesterol and diosgenin) are under different regulatory mechanisms. Cholesterol is involved in a number of biological processes (including membrane permeability) and other regulatory mechanism can overlap to maintain homeostasis.

Several studies that exploited transcriptome sequencing can be referred to diosgenin biosynthesis. Sequencing of fenugreek and Asparagus racemosus transcriptomes (Vaidya et al. 2013; Upadhyay et al. 2014) suggested that saponins were formed from sitosterol via cycloartenol. In asparagus, $C Y P 51$ and $D W F 1$ genes were overexpressed after MeJ treatment. Five genes ( $Q Q E, C A S, C Y P 51$, $D W F 5$, and $D W F 1$ ) involved in the biosynthesis of phytosterols were found in Dioscorea composite (Wang et al. 2015). These genes were overexpressed in the leaves and tubers as compared with the stem and shoot. However, the relative level of expression exceeded the value of 1 only for CAS and CYP51 genes.

\section{Identification and expression analysis of candidate genes involved in steroidal sapogenin biosynthesis}

Steroid pathway is the last step of steroidal sapogenin biosynthesis. There are two ways of diosgenin biosynthesis from 2,3-oxidosqualene: the first starts from lanosterol via the formation of cholesterol and the second starts from cycloartenol via sitosterol (Vaidya et al. 2013; Upadhyay et al. 2014). Phytosterols are synthesized from cycloartenol; however, the steps at which the biosynthesis of steroidal saponins and phytosterol diverges have not been elucidated (Faizal and Geelen 2013). The transcriptomes annotated within our experiment revealed multiple transcripts encoding all known enzymes involved in the steroid biosynthesis pathway. Based on the KEGG results, we found 10, 11, and 15 unigenes involved in steroid biosynthesis in RDA-CHL, RDA-MeJ, and RDA-SQ libraries, respectively. However, based on NR annotations, a number of unigenes were identified as "unknown," "uncharacterized," or "hypothetical protein," although they possessed suitable domains, i.e., PF00432.16 (prenyltransferases and squalene oxidases) or PF01073.14 ( $3 \beta$ hydroxysteroid dehydrogenases), that can be accounted to database of unigenes potentially involved in sterol biosynthesis. Some of these transcripts showed increased FPKM values, thus indicating enhanced expression. We annotated unigenes involved both in biosynthesis via a cholesterol and sitosterol, but at the similarities above $60 \%$ no transcripts corresponding to lanosterol synthase (EC:5.4.99.7) and 3-ketosteroid reductase (KR; EC:1.1.1.270) were found in RDA products (Table S8).

We identified unigene comp20077 (RDA-CHL) annotated as cytochrome P450 90B1-like, and contribution of this enzyme to steroidal sapogenin synthesis pathway can be considered. In brassinosteroid biosynthesis, C-22 steroid hydroxylase (CYP90B1) catalyzes hydroxylation of C-22. Furthermore, overexpression of CYP90B1 in Escherichia coli showed broad catalytic activity with respect to the sterols containing 27- (cholesterol, cholestanol), 28(campesterol, campestanol), and 29-carbon atoms (sitosterol). CYP90B1 preferred plant sterols with carbon atoms C5-C6 having a double bond to stanols that contained no double bond. Recombinant CYP90B1 showed the highest catalytic activity in hydroxylation of cholesterol to 22-hydroxycholesterol (Fujita et al. 2006).

As regards diosgenin biosynthesis process, we hypothesized that ring $\mathrm{E}$ closure was created as a result of hemiketal reaction with $-\mathrm{OH}$ group in $\mathrm{C}-16$ and $=\mathrm{O}$ group in $\mathrm{C}-22$. The keto group may be formed by oxidized hydroxyl group. We supposed that oxidation in C-16 might be catalyzed by unspecific monooxygenase EC:1.14.14.1. This enzyme takes part in a number of reactions, i.e., oxidation of estrone to 16 -hydroxyestrone; estradiol-17 $\beta$ to estriol; dehydroepiandrosterone to 16 $\alpha$-hydroxydehydroepiandrosterone. We found six unigenes for RDA-CHL, seven unigenes for RDA-MeJ, and six unigenes for RDASQ that were annotated as cytochrome p450 EC:1.14.14.1 (Table S9). Especially for RDA-SQ, four of the six unigenes had high values of FPKM (from 329.80 to 4809.92).

The next stages of brassinosteroid biosynthesis involve cytochrome CYP734A1 with 26-hydroxylase activity that converts brassinolide and castasterone into their 26-hydroxy derivatives (Turk et al. 2003). We annotated four unigenes for RDA-CHL (comp3187, comp4513, comp51591, comp73533), two unigenes for RDA-MeJ (com$\mathrm{p} 2112$, comp2587), and four unigenes for RDA-SQ (comp101982, comp3589, comp4033, comp51388). These unigenes could also be involved in steroidal sapogenin biosynthesis. Some of them had raised FPKM values (comp4513 1241.42; comp3589 1107.27; comp4033 785.41).

Within differentially expressed transcripts, we annotated also cytochrome $\mathrm{P} 450$ with 26-hydroxylase activity orthologous with CYP18A family (KEGG K14985) that can be involved in diosgenin biosynthesis. CYP18A1 enzyme takes part in insect hormone biosynthesis, 
catalyzes hydroxylation of 20-hydroxyecdysone to 20,26dihydroxyecdysone, and is a key enzyme of Drosophila steroid hormone inactivation. We found two corresponding unigenes, comp6079 (RDA-CHL) and comp2698 (RDAMeJ) with high FPKM values (3264.61 for comp6079 and 453.18 for comp2698), which confirmed overexpression of these transcripts and suggested the role of the corresponding enzyme in hydroxylation of C-26.

Ecdysteroids, present mainly in insects and other invertebrates, were found also in many plant species (more than 300 different phytoecdysteroids). Although ecdysteroids originate from sterols, their biosynthesis and intermediates have not been established yet. For example, in fern 22- and 25-hydroxy derivatives of cholesterol were converted to ecdysone and 22-hydroxyecdysone (Dinan et al. 2009). The presence of ecdysteroids in plants and overexpression of unigenes orthologous to CYP18A in the analyzed samples led to the conclusion that this cytochrome might recognize a structure of the sterol and participate in the biosynthesis of diosgenin.

Further enzymes and reactions resulting in diosgenin biosynthesis are unknown but cytochromes may play a significant role. After oxidation and hydroxylation of carbon $\mathrm{C} 16, \mathrm{C} 22$, and $\mathrm{C} 26$ and creation of a hemiketal form, the next step is to create the F ring by converting furostan form (aliphatic chain) into spirostan (ring F). Closing aliphatic chain to form ring $\mathrm{F}$ in spirostan requires both 26- $O$ glucoside and $-\mathrm{OH}$ group in $\mathrm{C}-22$. An addition of glucose molecule to a hydroxyl group in C-26 (glycosylation) is probably catalyzed by sterol glucosyltransferase. Glucose is transferred from uridine diphosphate glucose (UDPglucose); therefore, the enzyme is UDP-glucosyltransferase (2.4.1.-) (Kalinowska et al. 2005). Furostanol saponins are usually present as $26-O$-glucosides and activity of specific $26-O-\beta$-glucosidases that eliminate glucose results in the creation of F ring (Moreau et al. 2002). Protodioscin 26- $O$ $\beta$-D-glucosidase (EC:3.2.1.186) that catalyzes a cleavage of glucose from protodioscin and formation of 26-deglucoprotodioscin was characterized in Solanum torvum. In fenugreek transcripts selected upon MeJ treatment, we found two unigenes showing high similarity to protodioscin $26-O$ - $\beta$-D-glucosidase at protein level. Sequences comp17664 (alignment 33\% e value $6 e-09$ ) and comp91232 (52\% alignment $e$ value 7e-07) were annotated as $\beta$-glucosidases (EC:3.2.1.21).

\section{Mechanism of diosgenin biosynthesis from cycloartenol via cholesterol}

The annotated unigenes were used to study a secondary metabolic pathway. We focused on the genes and enzymes that could be involved in steroidal sapogenin biosynthesis. Terpenoid backbone is synthesized from mevalonate pathway leading to 2,3-oxidosqualene. Our results suggested that cholesterol was synthesized from cycloartenol. Diener et al. (2000) proposed a pathway leading from cycloartenol to cholesterol and its 24-methyl and 24-ethyl derivatives. We proposed a similar pathway, where double bond in $\mathrm{C} 24$ was maintained to the stage when desmosterol was reduced to cholesterol. Biosynthesis of cholesterol from cycloartenol in plants was also recently proposed by Talapatra and Talapatra (2015). Our hypothesis was further confirmed by a lack of transcripts corresponding to lanosterol synthase-an enzyme essential in this biosynthesis beginning from lanosterol in animals and fungi.

Cycloartenol synthase (CAS) and lanosterol synthase have similar (77-79\%) amino acid sequences in Arabidopsis thaliana (Suzuki et al. 2006; Umate 2015). In sequenced subtraction libraries, only two unigenes (comp99 and comp166794) were annotated as CAS and no sequence corresponding to lanosterol synthase was identified. The two sequences comp99 and comp166794 were aligned using blastx and showed 85 and $98 \%$ identity to cycloartenol synthase from Arabidopsis thaliana and Medicago truncatula, respectively. We also found $60 \%$ homology of these sequences to lanosterol synthase from A. thaliana (LAS1, At3g45130), while no gene annotated as lanosterol synthase was identified for M. truncatula.

We propose a pathway of cholesterol biosynthesis (Fig. 4) that involves the same enzymes that catalyze phytosterol biosynthesis, except for sterol methyltransferases SMT1 and SMT2. Cycloartenol is formed from 2,3oxidosqualene by cycloartenol synthase (5.4.99.8) and the next step-methylation of C-24 is skipped. In cycloartenol, one methyl group from C-4 is removed by sterol- $4 \alpha$-methyl oxidase (SMO), 3- $\beta$-hydroxysteroid- $4 \alpha$-carboxylate 3 -dehydrogenase (HSD), and 3-ketosteroid reductase (KR). Then, CPI isomerizes the cyclopropane ring in 29-norcycloartenol to form $\Delta^{8}$ tetracycles present in the tetracyclic sterols. The next step is demethylation of C-14 and reduction of the resulting bond by CYP51 and FK, respectively. CYP51, a member of cytochrome P450 monooxygenase superfamily, is essential for sterol biosynthesis and it is assumed to be the only orthologous P450 family that exists in the fungi, mammal, and plant kingdom. HYD1 catalyzes a removal of a hydrogen from $7 \beta$ position and subsequent addition of a hydrogen at $9 \alpha$ position, reducing the existing $\Delta 8(9)$ double bond and producing $\Delta 7(8)$ double bond of the sterol $\mathrm{B}$ ring. Then, second demethylation step from C-4 is catalyzed by SMO, HSD, and KR. DWF7 is involved in $\Delta^{7}$ sterol C-5 desaturation step and DWF5 reduces the double bond in C-7. The last stage is a reduction of $\Delta^{24}$-bond of sterols and formation of cholesterol by DWF1.

The proposed pathway retains the order of changes occurring in the biosynthesis of phytosterols: methyl 
Fig. 4 Proposed pathway leading from cycloartenol to cholesterol in steroidal sapogenin biosynthesis. SMO sterol-4 $\alpha$-methyl oxidase (EC:1.14.13.72), comp60361 (XP_006358705.1), HSD 3 $\beta$ hydroxysteroid- $4 \alpha$-carboxylate 3-dehydrogenase comp456, comp147036

(XP_003603241.1), comp27806 (XP_004501411.1), KR

3-ketosteroid reductase, $C P I$ cycloeucalenol cycloisomerase (EC:5.5.1.9) comp1007, comp3693, comp50967 (XP_003602088.1), comp230 (XP_004500654.1), CYP51 sterol 14-demethylase (EC:1.14.13.70) comp4337, comp2760, comp4118 (ABC59074.1), FK $\Delta$ 14-sterol reductase (EC:1.3.1.70) comp3967, comp21207, comp2560 (XP_004497600.1), HYD1 $\Delta^{8}-\Delta^{7}$-sterol isomerase (EC:5.3.3.5) comp71327 (XP_003534176.2), DWF7 $\Delta^{7}$ sterol-C5-desaturase (EC:1.14.21.6) comp60818 (XP_003849939.1), DWF5

7-dehydrocholesterol reductase/ sterol $\Delta^{7}$-reductase

(EC:1.3.1.21) comp4188, comp3298, comp2096, comp3395, (XP_004507556.1), $D W F 1$ sterol $\Delta^{\overline{24}}$-reductase (EC:1.3.1.72) comp4546, comp2786, comp3715 (P93472.1)

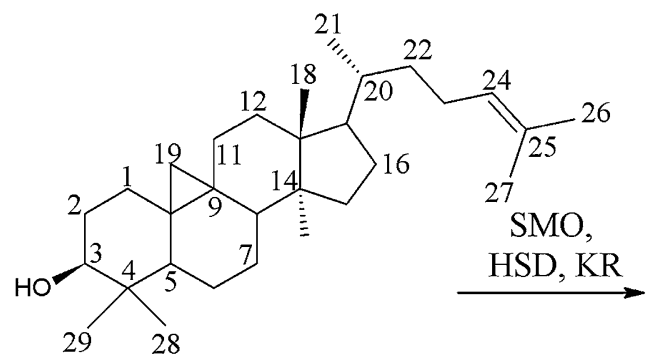

cycloartenol<smiles>CC(C)=CCC[C@@H](C)C1CC=C2C3=C(CC[C@]21C)[C@@]1(C)CC[C@H](O)[C@H](C)C1CC3</smiles>

4 $\alpha$-methylcholesta-8,14,24-trien-3 $\beta$-ol

CYP51

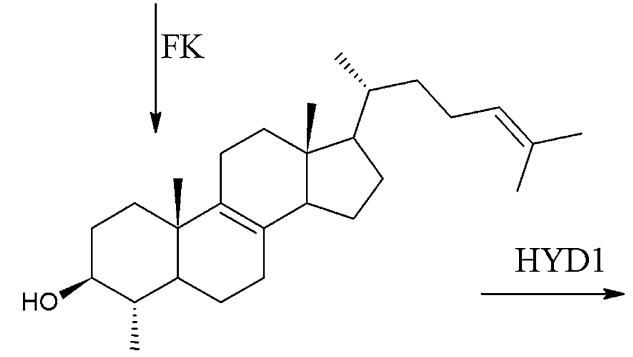

$4 \alpha$-methylcholesta-8,24-dien-3 $\beta$-ol<smiles>CCC1CCC2C3=CC=C4C[C@@H](O)CC[C@]4(C)C3CC[C@]12C</smiles>

7-dehydrodesmosterol

DWF7<smiles>CC(C)=CCC[C@@H](C)C1CCC2C3CC=C4C[C@@H](O)CC[C@]4(C)C3CC[C@]21C</smiles>

desmosterol<smiles></smiles><smiles>CC(C)=CCC[C@@H](C)C1CC[C@]2(C)C3=C(CC[C@]12C)[C@@]1(C)CC[C@H](O)[C@H](C)C1CC3</smiles>

29-norlanosterol<smiles>CC(C)=CCC[C@@H](C)C1CCC2C3=CCC4[C@@H](C)[C@@H](O)CC[C@]4(C)C3CC[C@@]21C</smiles>

4 $\alpha$-methylcholesta-7,24-dien-3 $\beta$-ol $\mathrm{SMO}$
$\mathrm{HSD}, \mathrm{KR}$

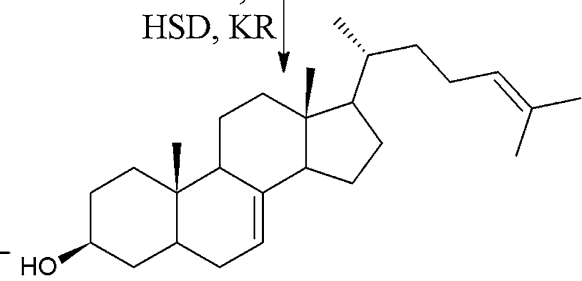
cholesta-7,24-dien-3 $\beta$-ol<smiles>CC(C)CCC[C@@H](C)C1CCC2C3CC=C4C[C@@H](O)CC[C@]4(C)C3CC[C@@]21C</smiles>
cholesterol groups are removed in the order of $\mathrm{C} 4, \mathrm{C} 14$, and $\mathrm{C} 4$, and there is a close cooperation of three enzymes (SMO, HSD, and KR) catalyzing two reactions of demethylation from C4 (Rahier 2011) and sequential reactions of C14 reduction, and the double bond isomerization of $\mathrm{C} 8$ to $\mathrm{C} 7$ (Schrick et al. 2002).
For the first time, we proposed the enzymes that are involved in further processing of cholesterol (Fig. 5). We propose that in this process unspecific monooxygenase EC:1.14.14.1 participates in hydroxylation of C-16, and CYP18A1/CYP734A1 catalyzes hydroxylation of C-26 and probably precedes the reaction of glucose addition to 
<smiles>CC(C)CCC(O)[C@H](C)C1CCC2C3CC=C4CC(O)CC[C@]4(C)C3CC[C@@]21C</smiles>

cholesterol

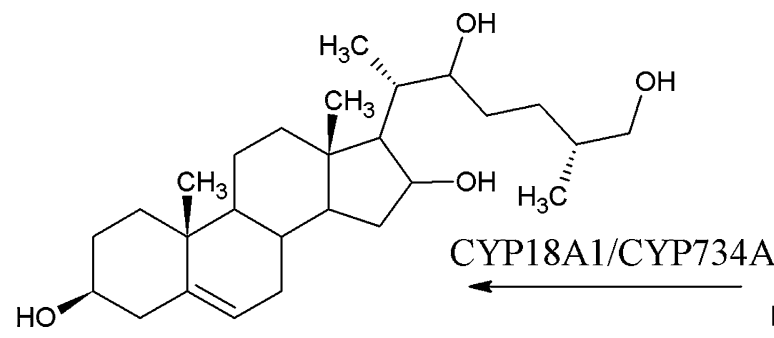

(3ß,25R)-cholesta-5-en-3,16,22,26-tetraol<smiles>C[C@H](CO)CCC(=O)[C@H](C)C1C(O)CC2C3CC=C4C[C@@H](O)CC[C@]4(C)C3CC[C@]21C</smiles>

(3ß,25R)-3,16,26-trihydroxycholesta-5-en-22-one (3ß)-cholesta-5-en-3,22-diol

EC:1.14.14.1

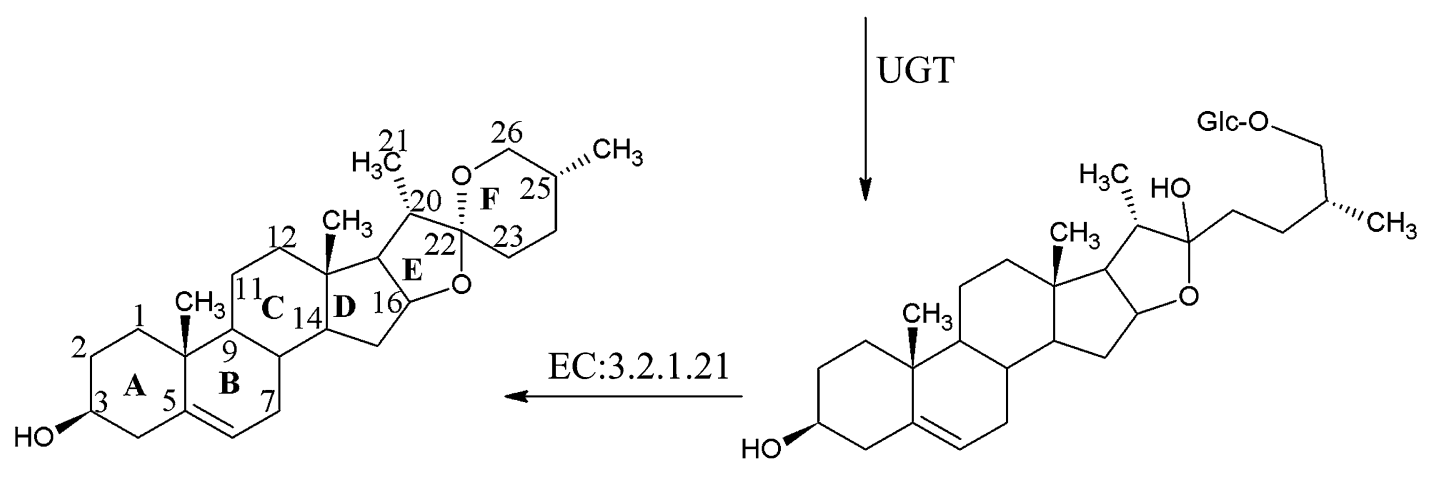

diosgenin

25R-spirost-5-en-3ß-ol
Fig. 5 Proposed pathway leading form cholesterol to diosgenin. CYP90B1 steroid 22 $\alpha$-hydroxylase comp20077 (XP_003629467.1), EC:1.14.14.1 unspecific monooxygenase comp35839, comp88342, comp32891 (XP_003610974.1), CYP18A1/CYP734A1 cytochromes that catalyze C-26 hydroxylation comp6079 (XP_003615674.1),

cholesterol. Moreover, qRT-PCR data suggest that hydroxylation of C-22 may be catalyzed by steroid $22 \alpha-$ hydroxylase (CYP90B1). The last two stages involve the comp2698 (XP_007036488.1), comp4513, comp2112, comp3589 (XP_004495852.1), UGT UDP-glucosyltransferase comp2754 (XP_008085517.1), EC:3.2.1.21 $\beta$-glucosidase comp1334, comp17664, comp2749 (XP_006575592.1)

closure of the F ring and formation of spiroketal. First, the glycosylation of C26 is catalyzed by UDP-glucosyltransferase, and then glucose molecule is removed by $\beta$ - 
glucosidase (EC:3.2.1.21) to form a spiro ring of diosgenin. The order of carbon hydroxylation of C16, C22, and C26 needs to be confirmed experimentally. Alternative pathways of diosgenin biosynthesis in fenugreek induced by inhibitors or resulting from genetic diversity may also be feasible.

\section{Conclusions}

We reported on the application of next-generation sequencing (NGS) technology for the analysis of cDNARDA products after treatment with methyl jasmonate, cholesterol, and squalene. Subtraction procedure equilibrated the frequencies of transcripts and increased a chance to identify unique genes. De novo assembly and functional annotations were performed. As a result of the analysis of related pathways, we identified the majority of candidate genes involved in important secondary metabolic pathways as sterols and steroidal sapogenins. Moreover, we propose a biosynthetic pathway leading to formation of diosgenin from cycloartenol via cholesterol.

Author contribution statement JC collected the literature, prepared plant material, performed UPLC analysis, participated in RNA isolation, RDA procedure and sequencing, interpreted scientific information, and wrote the manuscript. MS participated in RNA isolation and RDA procedure and helped in data interpretation. MG participated in RDA procedure, library preparation, and sequencing. MT coordinated the experiment, was involved in scientific advising, and helped in the manuscript edition.

Acknowledgements The study was supported by EU Regional Operational Program project UDA-RPPK.01.03.00-18-018/13-0 "The use of molecular and proteomic tools for identification of genes and enzymes with potential in biotechnology."

\section{Compliance with ethical standards}

Conflict of interest The authors declare that they have no conflict of interest.

Open Access This article is distributed under the terms of the Creative Commons Attribution 4.0 International License (http://crea tivecommons.org/licenses/by/4.0/), which permits unrestricted use, distribution, and reproduction in any medium, provided you give appropriate credit to the original author(s) and the source, provide a link to the Creative Commons license, and indicate if changes were made.

\section{References}

Aasim M, Khawar KM, Yalcin G, Bakhsh A (2014) Current trends in fenugreek biotechnology and approaches towards its improvement. Am J Social Issues Humanities (AJSIH), Special Issue, pp 128-136

Annadurai RS, Jayakumar V, Mugasimangalam RC, Katta M, Anand S, Gopinathan S, Sarma SP, Fernandes SJ, Mullapudi N, Murugesan S, Rao SN (2012) Next generation sequencing and de novo transcriptome analysis of Costus pictus D. Don, a nonmodel plant with potent anti-diabetic properties. BMC Genom 13:663-678

Augustin JM, Kuzina V, Andersen SB, Bak S (2011) Molecular activities, biosynthesis and evolution of triterpenoid saponins. Phytochemistry 72:435-457

Benveniste P (2004) Biosynthesis and accumulation of sterols. Annu Rev Plant Biol 55:429-457

Bowler LD, Hubank M, Spratt BG (1999) Representational difference analysis of cDNA for the detection of differential gene expression in bacteria: development using a model of ironregulated gene expression in Neisseria meningitidis. Microbiology 145:3529-3537

Cao H, Nuruzzaman M, Xiu H, Huang J, Wu K, Chen X, Li J, Wang L, Jeong JH, Park SJ, Yang F, Luo J, Luo Z (2015) Transcriptome analysis of methyl jasmonate-elicited Panax ginseng adventitious roots to discover putative ginsenoside biosynthesis and transport genes. Int J Mol Sci 16:3035-3057

Chang Y (2003) Representational difference analysis. In: Ausubel FM, Brent R, Kingston RE, Moore DD, Seidman JG, Smith JA, Struhl K (eds) Current protocols in molecular biology. Wiley, Chichester, pp 1-12

Chappell J (2002) The genetics and molecular genetics of terpene and sterol origami. Curr Opin Plant Biol 5:151-157

Chaudhary S, Chikara SK, Sharma MC, Chaudhary A, Syed BA, Chaudhary PS, Mehta A, Patel M, Ghosh A, Iriti M (2015) Elicitation of diosgenin production in Trigonella foenum-graecum (fenugreek) seedlings by methyl jasmonate. Int J Mol Sci 16:29889-29899

Chikara SK, Pandey M, Pandey S, Vaidya K, Chaudhary S (2014) Next generation sequencing: a revolutionary tool for plant variety improvement. Am J Social Issues Humanities (AJSIH), Special Issue, pp 137-154

Conesa A, Götz S, Garcia-Gomez JM, Terol J, Talon M, Robles M (2005) Blast2GO: a universal tool for annotation, visualization and analysis in functional genomics research. Bioinformatics 21:3674-3676

De D, De B (2011) Elicitation of diosgenin production in Trigonella foenum-graecum L. seedlings by heavy metals and signaling molecules. Acta Physiol Plant 33:1585-1590

Dewick PM (2002) Medicinal natural products: a biosynthetic approach, 2nd edn. Wiley, Chichester

Diener AC, Li H, Zhou W, Whoriskey WJ, Nes WD, Fink GR (2000) STEROL METHYLTRANSFERASE 1 controls the level of cholesterol in plants. Plant Cell 12:853-870

Dinan L, Harmatha J, Volodin V, Lafont R (2009) Phytoecdysteroids: diversity, biosynthesis and distribution. In: Smagghe G (ed) Ecdysone: structures and functions. Springer Science + Business Media BV, Dordrecht, pp 3-45

Faizal A, Geelen D (2013) Saponins and their role in biological processes in plants. Phytochem Rev 12:877-893

Fujita S, Ohnishi T, Watanabe B, Yokota T, Takatsuto S, Fujioka S, Yoshida S, Sakata K, Mizutani M (2006) Arabidopsis CYP90B1 catalyses the early C-22 hydroxylation of C27, C28 and C29 sterols. Plant J 45(5):765-774

Grabherr MG, Haas BJ, Yassour M, Levin JZ, Thompson DA, Amit I, Adiconis X, Fan L, Raychowdhury R, Zeng Q et al (2011) Fulllength transcriptome assembly from RNA-Seq data without a reference genome. Nat Biotechnol 29:644-652. doi:10.1038/nbt. 1883 
Hubank M, Schatz DG (1994) Identifying differences in mRNA expression by representational difference analysis of cDNA. Nucleic Acids Res 22:5640-5648

Hubank M, Shatz DG (1999) cDNA representational difference analysis: a sensitive and flexible method for the identification of differentially expressed genes. Method Enzymol 303:325-349

Hwang HS, Lee H, Choi YE (2015) Transcriptomic analysis of Siberian ginseng (Eleutherococcus senticosus) to discover genes involved in saponin biosynthesis. BMC Genom 16:180-192

Kalinowska M, Zimowski J, Pączkowski C, Wojciechowski ZA (2005) The formation of sugar chains in triterpenoid saponins and glycoalkaloids. Phytochem Rev 4:237-257

Kornobis E, Cabellos L, Aguilar F, Frias-Lopez C, Rozas J, Marco J, Zardoya R (2015) TRUFA: a user-friendly web server for de novo RNA-seq analysis using cluster computing. Evol Bioinform 11:97-104

Lambert E, Faizal A, Geelen D (2011) Modulation of triterpene saponin production: in vitro cultures, elicitation, and metabolic engineering. Appl Biochem Biotechnol 164:220-237

Langmead B, Salzberg S (2012) Fast gapped-read alignment with Bowtie 2. Nat Methods 9:357-359

Leite GGS, Baeza LC, Batista AA, Barbosa MIF, Pavan FR, Leite CQF, Silva JL, Hirata RDC, Hirata MH, Cardoso RF (2013) Genes differentially expressed by Mycobacterium tuberculosis after exposure to ruthenium phosphinic compound and isoniazid. Int J Microbiol Res 5:356-361

Livak KJ, Schmittgen TD (2001) Analysis of relative gene expression data using real-time quantitative PCR and the 2(-Delta Delta $\mathrm{C}(\mathrm{T})$ ) method. Methods 25(4):402-408

Mehrafarin A, Qaderi A, Rezazadeh S, Naghdi BH, Noormohammadi G, Zand E (2010) Bioengineering of important secondary metabolites and metabolic pathways in fenugreek (Trigonella foenum-graecum L.). J Med Plants 9(35):1-18

Mehrafarin A, Rezazadeh Sh, Naghdi Badi H, Noormohammadi Gh, Zand E, Qaderi A (2011) A review on biology, cultivation and biotechnology of fenugreek (Trigonella foenum-graecum L.) as a valuable medicinal plant and multipurpose. J Med Plants 10:6-24

Moreau RA, Whitaker BD, Hicks KB (2002) Phytosterols, phytostanols, and their conjugates in foods: structural diversity, quantitative analysis, and health-promoting uses. Prog Lipid Res 41:457-500

Moriya Y, Itoh M, Okuda S, Yoshizawa A, Kaneshia M (2007) KASS: an automatic genome annotation and pathway reconstruction server. Nucleic Acids Res 35:W182-W185

Murashige T, Skoog F (1962) A revised medium for rapid growth and bioassays with tobacco tissue cultures. Physiol Plant 15:473-497

Patel K, Gadewar M, Tahilyani V, Patel DK (2012) A review on pharmacological and analytical aspects of diosgenin: a concise report. Nat Prod Bioprospect 2:46-52

Patel SS, Shah DB, Panchal HJ (2014) De novo RNA seq assembly and annotation of Trigonella foenum-graecum L. (SRR066197). Legume Genomics Genet 5(7):1-7

Petropoulos GA (2002) Fenugreek-the genus Trigonella. Taylor and Francis, London

Rahier A (2011) Dissecting the sterol C-4 demethylation process in higher plants. From structures and genes to catalytic mechanism. Steroids 76:340-352

Raju J, Rao CV (2012) Diosgenin, a steroid saponin constituent of yams and fenugreek: emerging evidence for applications in medicine. In: Rasooli I (ed) Bioactive compounds in phytomedicine. InTech, pp 125-142. doi:10.5772/26700

Sangwan RS, Tripathi S, Singh J, Narnoliya LK, Sangwan NS (2013) De novo sequencing and assembly of Centella asiatica leaf transcriptome for mapping of structural, functional and regulatory genes with special reference to secondary metabolism. Gene 525:58-76

Savikin-Fodulovic K, Grubisic D, Culafic L, Menkovic N, Ristic M (1998) Diosgenin and phytosterols content in five callus lines of Dioscorea balcanica. Plant Sci 135:63-67

Schrick K, Mayer U, Martin G, Bellini C, Kuhnt C, Schmidt J, Jürgens $G$ (2002) Interactions between sterol biosynthesis genes in embryonic development of Arabidopsis. Plant J 31:61-73

Shi CY, Yang H, Wei CL, Yu O, Zhang ZZ, Jiang CJ, Sun J, Li YY, Chen Q, Xia T, Wan XC (2011) Deep sequencing of the Camellia sinensis transcriptome revealed candidate genes for major metabolic pathways of tea-specific compounds. BMC Genom 12:131-150

Smetanska I (2008) Production of secondary metabolites using plant cell cultures. Adv Biochem Eng Biotechnol 111:187-228

Snehlata HS, Payal DR (2012) Fenugreek (Trigonella foenumgraecum L.): an overview. Int J Current Pharm Rev Res 2(4):169-187

Suzuki M, Xiang T, Ohyama K, Seki H, Saito K, Muranaka T, Hayashi H, Katsube Y, Kushiro T, Shibuya M, Ebizuka Y (2006) Lanosterol synthase in dicotyledonous plants. Plant Cell Physiol 47(5):565-571

Szakiel A, Pączkowski C, Henry M (2011) Influence of environmental abiotic factors on the content of saponins in plants. Phytochemistry 10:471-491

Talapatra SK, Talapatra B (2015) Chemistry of plant natural products: stereochemistry, conformation, synthesis, biology and medicine. Springer, Berlin

Turk EM, Fujioka S, Seto H, Shimada Y, Takatsuto S, Yoshida S, Denzel MA, Torres QI, Neff MM (2003) CYP72B1 inactivates brassinosteroid hormones: an intersection between photomorphogenesis and plant steroid signal transduction. Plant Physiol 133(4):1643-1653

Umate P (2015) Comparison of genes encoding enzymes of sterol biosynthesis from plants to orthologs in yeast. J Rice Res 4:160-171

Upadhyay S, Phukan UJ, Mishra S, Shukla RK (2014) De novo leaf and root transcriptome analysis identified novel genes involved in steroidal sapogenin biosynthesis in Asparagus racemosus. BMC Genom 15:746-759

Vaidya K, Ghosh A, Kumar V, Chaudhary S, Srivastava N, Katudia K, Tiwari T, Chikara SK (2013) De novo transcriptome sequencing in Trigonella foenum-graecum L. to identify genes involved in the biosynthesis of diosgenin. Plant Genome 6:1-11

Vincken JP, Heng L, de Groot A, Gruppen H (2007) Saponins, classification and occurrence in the plant kingdom. Phytochemistry 68:275-297

Wang X, Chen D, Wang Y, Xie J (2015) De novo transcriptome assembly and the putative biosynthetic pathway of steroidal sapogenins of Dioscorea composita. PLoS One 10(4):e0124560

Wu S, Zhu Z, Fu L, Niu B, Li W (2011) WebMGA: a customizable web server for fast metagenomic sequence analysis. BMC Genom 12:444-453

Xue Z, Duan L, Liu D, Guo J, Ge S, Dicks J, ÓMáille P, Osbourn A, Qi X (2012) Divergent evolution of oxidosqualene cyclases in plants. New Phytol 193:1022-1038

Ye J, Coulouris G, Zaretskaya I, Cutcutache I, Rozen S, Madden TL (2012) Primer-BLAST: a tool to design target-specific primers for polymerase chain reaction. BMC Bioinform 13:134-145

Zhang HB, Xia EH, Huang H, Jiang JJ, Liu BY, Gao LZ (2015) De novo transcriptome assembly of the wild relative of tea tree (Camellia taliensis) and comparative analysis with tea transcriptome identified putative genes associated with tea quality and stress response. BMC Genom 16:298-312 